\title{
Group A streptococcal bacteremias in Southwest Finland 2007-2018: epidemiology and role of infectious diseases consultation in antibiotic treatment selection
}

\author{
Johanna Vilhonen $^{1}$ (D) Jaana Vuopio ${ }^{2} \cdot$ Tero Vahlberg $^{3} \cdot$ Kirsi Gröndahl-Yli-Hannuksela $^{4} \cdot$ Kaisu Rantakokko-Jalava $^{5}$. \\ Jarmo Oksi ${ }^{6}$
}

Received: 20 December 2019 / Accepted: 11 February 2020 / Published online: 25 February 2020

(C) The Author(s) 2020

\begin{abstract}
The incidence of invasive group A streptococcal (GAS) infections has shown a fluctuating but increasing trend in Finland. The impact of infectious diseases specialist consultation (IDSC) on the antimicrobial therapy of GAS bacteremia has not been studied earlier. A retrospective study on adult GAS bacteremia in The Hospital District of Southwest Finland (HDSWF) was conducted from 2007 to 2018. Data on incidence of bacteremic GAS cases were gathered from the National Infectious Disease Register. Clinical data were obtained by reviewing the electronic patient records. The overall incidence of GAS bacteremia in HDSWF was 3.52/100,000, but year-to-year variation was observed with the highest incidence of 7.93/100,000 in 2018. A total of 212 adult GAS bacteremia cases were included. A record of IDSC was found $(+)$ in $117(55.2 \%)$ cases, not found (-) in $71(33.5 \%)$ cases and data were not available in $24(11.3 \%)$ cases. Among IDSC+ cases, $57.3 \%$ were on penicillin G treatment whereas in the group IDSC - only $22.5 \%$, respectively $(\mathrm{OR}=4.61$, 95\% CI 2.37-8.97; $p<0.001)$. The use of clindamycin as adjunctive antibiotic was more common among IDSC+ $(54.7 \%)$ than IDSC $-(21.7 \%)(\mathrm{OR}=4.51,95 \%$ CI $2.29-8.87 ; p<0.001)$. There was an increasing trend in incidence of GAS bacteremia during the study period. Narrow-spectrum beta-lactam antibiotics were chosen, and adjunctive clindamycin was more commonly used, if IDSC took place. This highlights the importance of availability of IDSC but calls for improved practice among infectious diseases specialists by avoiding combination therapy with clindamycin in nonsevere invasive GAS infections.
\end{abstract}

Keywords Group A streptococcus $\cdot$ Epidemiology $\cdot$ Antibiotic treatment $\cdot$ Infectious diseases specialist consultation

Johanna Vilhonen

jhhjun@utu.fi

1 Department of Infectious Diseases, Turku University Hospital; Doctoral Programme in Clinical Research (DPCR), University of Turku, Turku, Finland

2 Institute of Biomedicine, University of Turku; Department of Clinical Microbiology, Turku University Hospital, Turku, Finland

3 Department of Clinical Medicine, Biostatistics, University of Turku, Turku, Finland

4 Institute of Biomedicine, University of Turku, Turku, Finland

5 Department of Clinical Microbiology, Turku University Hospital, Turku, Finland

6 Department of Infectious Diseases, Turku University Hospital, University of Turku, Turku, Finland

\section{Introduction}

Streptococcus pyogenes (group A streptococcus, GAS) is a well-recognized human pathogen that causes commonly non-invasive infections such as pharyngitis and nonnecrotizing cellulitis but can also cause invasive infections (iGAS) such as bacteremia and severe iGAS infections, e.g., streptococcal toxic shock syndrome (STSS) and necrotizing fasciitis (NF), respectively. GAS infections are associated with remarkable morbidity and mortality worldwide with an estimated 500,000 deaths per year [1,2].

The incidence of iGAS infections is known to fluctuate. In Finland, an increasing trend in the overall incidence has been observed since 1995 [3-5]. Concordant observations are reported also from other countries [6-8].

GAS isolates have remained universally susceptible to penicillin and penicillin-resistant strains have not been reported to 
date. However, Vannice et al. reported recently clinical GAS isolates with a $p b p 2 x$ missense mutation and elevated minimum inhibitory concentrations for ampicillin, amoxicillin and cefotaxime [9]. Similarly, GAS strains with lowered susceptibility to beta-lactams have been reported in a large multicountry study, including Finland [10]. Beta-lactam antibiotics, and especially penicillin, are the basis of the antimicrobial therapy of GAS infections. Concurrent therapy with clindamycin is observed to reduce mortality in severe iGAS infections [11-13].

The Hospital District of Southwest Finland (HDSWF) has a total of over 470,000 residents and 5 hospitals (Turku University Hospital and regional hospitals in Salo, Loimaa, Uusikaupunki, and Turku). The aim of the study is to report incidence, emm type distribution, clinical pictures and outcome of GAS bacteremias in the HDSWF during 20072018. In addition, our purpose is to assess the effect of an infectious diseases specialist consultation (IDSC) on the antimicrobial therapy of GAS bacteremia during this study period.

\section{Materials and methods}

\section{Data collection}

Since 1995, invasive GAS infections (only isolations from blood and cerebrospinal fluid) have been notifiable in Finland according to the Communicable Diseases Act. The diagnosing laboratory reports GAS bacteremias and isolations from cerebrospinal fluid to the National Infectious Disease Register (NIDR) maintained by the Finnish institute of health and welfare (THL). The data of each iGAS case are recorded in the NIDR to the hospital district that the patient belongs to according to their place of domicile. Data on incidence of bacteremic GAS cases were gathered from NIDR [14].

The Department of Hospital Hygiene and Infection Control in the HDSWF maintains a database (SAI) to which all the microbial findings in clinical samples, e.g., bacteremias, are recorded with the national identity code of the patient and the date of specimen. The SAI database records all iGAS cases treated in HDSWF irrespective of patient's place of domicile. All adult bacteremic GAS cases treated in the hospitals of HDSWF from Jan 2007 until Dec 2018 were retrospectively identified from SAI-register. The cases meeting the inclusion criteria were included in this study, and their electronic patient records were reviewed.

The electronic patient records were read by an infectious diseases specialist (JVi) and the data on patient demographics, underlying diseases, clinical course of bacteremia, infectious foci, antibiotic therapy, a record of IDSC and outcome were documented.

\section{Inclusion criteria}

The inclusion criteria were at least one positive blood culture for GAS with concurrent clinical signs of infection, age of $\geq 18$ years and at least one contact to any of the hospitals of the HDSWF during the GAS bacteremia episode.

\section{emm typing}

THL has emm-typed invasive GAS strains since 2005, and data on emm types were acquired from NIDR. In altogether, 10 cases the data on emm types were not available from NIDR. For 8 cases of these 10, the isolate was obtained from the culture collection of Turku University Hospital and typed at the University of Turku. Emm typing was performed according to the guidelines of the Centers for Disease Control and Prevention (CDC) [15].

\section{Definitions}

The underlying diseases were classified according to the Charlson Comorbidity Index (CCI). The CCI was further divided into four categories according to the Charlson's original study, 0 score is $0,1-2$ scores is $1,3-4$ scores is 2 and $\geq 5$ is 3 [16].

Healthcare-acquired GAS bacteremia was defined as (1) bacteremia with positive blood cultures for GAS gathered $\geq$ $48 \mathrm{~h}$ after hospital admission or (2) GAS bacteremia associated with a healthcare-related procedure (including labor and previous hospital discharge) during 30 days before the positive blood cultures for GAS or (3) GAS bacteremia associated with foreign body surgery during 90 days before the positive blood cultures for GAS.

The definition of STSS was according to the case definition 2010 of CDC and included identification of GAS from a normally sterile site, hypotension and at least two other organ failures [17].

The infectious diseases specialist consultation was defined as a written note of IDSC in the patient record done by the treating doctor or infectious disease specialist during 5 days after the positive blood cultures for GAS. No available data on consultation (NADC) was used for cases who died during the $48 \mathrm{~h}$ after the positive blood cultures for GAS or who were transferred to a health care unit outside of HDSWF before the answer of blood cultures were available.

The first-line antibiotics and adjunctive antibiotics were defined as antibiotics given to the patient at the time when the first positive GAS blood culture result was available until the fifth day after taking the blood cultures. Beta-lactam antibiotics and vancomycin were regarded as first-line antibiotics and other antibiotics, if used, adjunctive antibiotics. 


\section{Statistical analyses}

Statistical analyses were performed with the IBM SPSS Statistics for Windows version 25 (IBM Corp., Armonk, $\mathrm{NY}$ ). A two-sided $p<0.05$ was considered statistically significant. Categorical data were analyzed by chi-square test, and mean age between IDSC groups was tested by two-sample test. The differences in antibiotic treatment selections between groups were analyzed by binary logistic regression. Poisson regression analysis was used to examine the change in incidence of GAS bacteremia in HDSWF during the 4-year follow-up periods (2007-2010, 2011-2014, and 2015-2018).

\section{Results}

\section{Epidemiology and clinical characteristics}

In total, 227 GAS bacteremia cases were identified from NIDR. The overall incidence of GAS bacteremia in HDSWF was $3.52 / 100,000$ person-years during the study period, but year-to-year variation was observed with the highest incidence of 7.93/100,000 person-years in 2018. Beside year 2018, high incidence values were also found in $2013(6.78 / 100,000$ person-years) and 2017 (6.05/100,000 person-years) whereas the incidence was lowest in 2010 (1.29/100,000 person-years) (Fig. 1). Incidences increased over 4-year follow-up periods between 2007 and 2010 (2.59/100,000 person-years), 20112014 (4.08/100,000 person-years) and 2015-2018 (5.34/ 100,000 person-years). The increase was significant in 2011-2014 (IRR $=1.58,95 \%$ CI $1.10-2.27, p=0.013)$ and 2015-2018 (IRR $=2.07,95 \%$ CI $1.47-2.91, p<0.001)$ compared with 2007-2010.

After analyzing the SAI data, a total of 212 adult GAS bacteremia cases met the inclusion criteria and were included in the study. The excluded cases were either under 18 years of age or had not been treated in any of the hospitals of the HDSWF. Clinical characteristics of the cases are summarized in Table 1 . The most common underlying condition was any atherosclerotic disease $20.8 \%$, but $22.2 \%$ of the cases had no underlying disease. Skin and soft tissue infections were the most common clinical manifestations (58.0\%). $21.7 \%$ of the cases were admitted to intensive care unit (ICU) and $15.6 \%$ met the STSS definition. Mortality among all cases in 7 days was $6.6 \%$ and in 90 days $13.2 \%$. Case fatality rate (CFR) in STSS was in 7 days $24.2 \%$ and in 90 days $27.3 \%$.

A record of IDSC was found (+) in $117(55.2 \%)$ cases, not found (-) in $71(33.5 \%)$ cases, while in $24(11.3 \%)$ cases, no data on consultation were available. The comparison of the groups IDSC+ and IDSC - is shown in Table 1. The patients with GAS bacteremia who received IDSC were more likely to be admitted to ICU, to have hypotension, STSS and disseminated intravascular coagulation (DIC) and to undergo any surgical intervention. The patients who did not receive IDSC were more likely to have diabetes mellitus and malignancies. Otherwise these two study groups did not differ statistically significantly. There was a slight trend toward lower mortality in IDSC+ group compared with IDSC - group even though it did not reach statistical significance.

\section{emm-type distribution}

The emm-type distribution of the bacteremic GAS isolates during the study period is summarized in Table 2 . The most prevalent emm types in the descending order were emm 28 (a total of 58 cases, $27.4 \%$ of all), emm 1 (42, 19.8\%), emm 89 (40, 18.9\%), emm 12 (18, 8.5\%), and emm 4 (14, 6.6\%). During the incidence peak years of 2013 and 2018, the most common emm types were emm 89 (12, 40.0\%) and emm 1 (17, $42.5 \%$ ). The increase in emm 1 isolate numbers was observed
Fig. 1 Annual number and incidence $/ 100,000 /$ person-years of bacteremic GAS cases in the HDSWF 2007-2018 as reported in NIDR

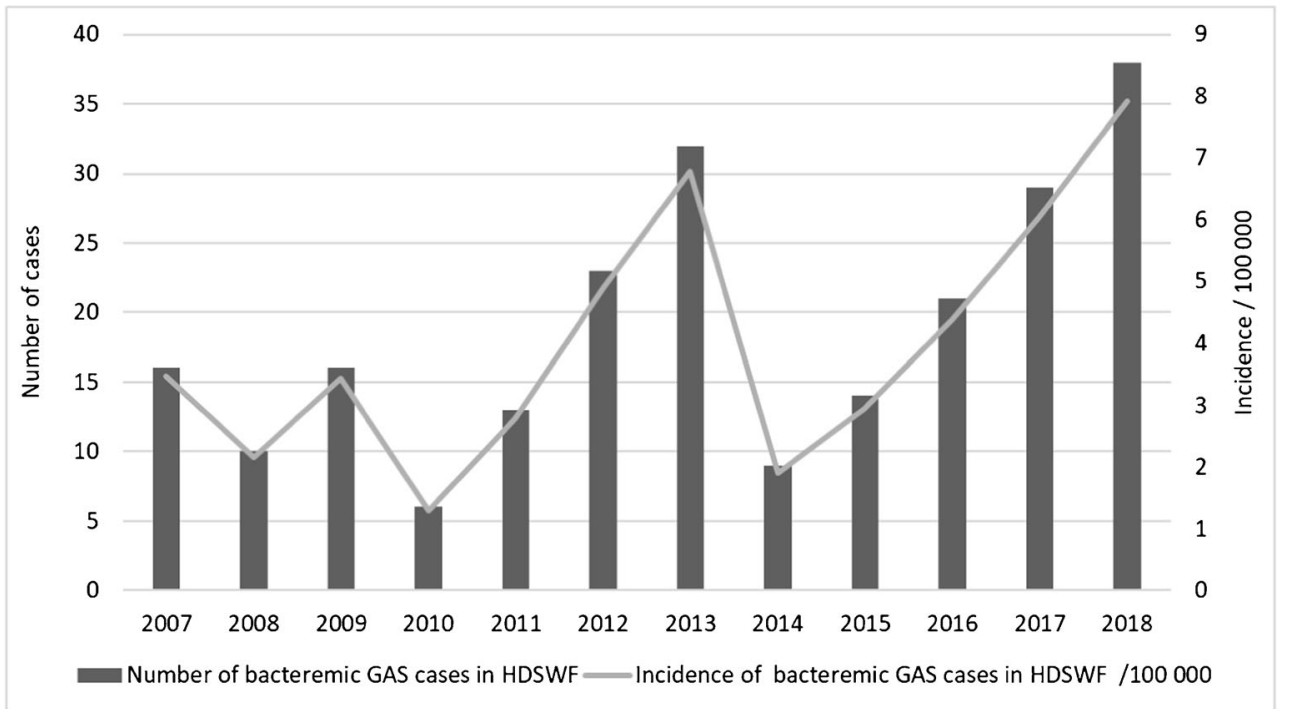


Table 1 Demographics and clinical characteristics of 212 patients with GAS bacteremia and comparison of patients with and without infectious diseases specialist consultation

\begin{tabular}{|c|c|c|c|c|c|}
\hline Variables & $\mathrm{IDSC}+(n=117)$ & IDSC $-(n=71)$ & $p$ value $^{\mathrm{a}}$ & $\operatorname{NADC}(n=24)$ & Total (212) \\
\hline Sex: male & $56(47.9)$ & $38(53.5)$ & 0.452 & $10(41.7)$ & $104(49.1)$ \\
\hline Age mean (SD) & $56.3(19.2)$ & $60.9(19.3)$ & 0.115 & $72.2(17.0)$ & $59.6(19.6)$ \\
\hline Healthcare-acquired & $19(16.2)$ & $9(12.7)$ & 0.506 & $3(12.5)$ & $31(14.6)$ \\
\hline Charlson class & & & 0.245 & & \\
\hline 0 & $36(30.8)$ & 14 (19.7) & & $1(4.2)$ & $51(24.1)$ \\
\hline 1 & 25 (21.4) & $15(21.1)$ & & $6(25.0)$ & $46(21.7)$ \\
\hline 2 & $20(17.1)$ & $11(15.5)$ & & $3(12.5)$ & $34(16.0)$ \\
\hline 3 & $36(30.8)$ & $31(43.7)$ & & $14(58.3)$ & $81(38.2)$ \\
\hline \multicolumn{6}{|l|}{ Underlying conditions } \\
\hline Diabetes mellitus & $13(11.1)$ & $16(22.5)$ & 0.036 & $6(25.0)$ & $35(16.5)$ \\
\hline${ }^{\mathrm{b}}$ Any atherosclerotic disease & $17(14.5)$ & $17(23.9)$ & 0.104 & $10(41.7)$ & $44(20.8)$ \\
\hline${ }^{\mathrm{c}}$ Chronic lung disease & $9(7.7)$ & $4(5.6)$ & 0.590 & $3(12.5)$ & $16(7.5)$ \\
\hline${ }^{\mathrm{d}}$ Alcohol abuse & $15(12.8)$ & $9(12.7))$ & 0.977 & $2(8.3)$ & $26(12.3)$ \\
\hline IVDU & $9(7.7)$ & $3(4.2)$ & 0.346 & $1(4.2)$ & $13(6.1)$ \\
\hline${ }^{\mathrm{e}}$ Immunosuppr. medication & $14(12.0)$ & $8(11.3)$ & 0.885 & $4(16.7)$ & $26(12.3)$ \\
\hline${ }^{\mathrm{f}}$ Any malignancy & $11(9.4)$ & $15(21.1)$ & 0.024 & $2(8.3)$ & $28(13.2)$ \\
\hline${ }^{\mathrm{g}}$ No underlying disease & $32(27.4)$ & $14(19.7)$ & 0.238 & $1(4.2)$ & $47(22.2)$ \\
\hline Allergy to penicillin & $3(2.6)$ & $4(5.6)$ & 0.281 & $1(4.2)$ & $8(3.8)$ \\
\hline \multicolumn{6}{|l|}{ Clinical manifestation } \\
\hline${ }^{\mathrm{h}} \mathrm{SSTI}$ & $67(57.3)$ & $41(57.7)$ & 0.948 & $15(62.5)$ & $123(58.0)$ \\
\hline${ }^{\mathrm{i}}$ Necrotizing fasciitis & $5(4.3)$ & $1(1.4)$ & 0.279 & $0(0)$ & $6(2.8)$ \\
\hline Pneumonia & $23(19.7)$ & $18(25.4)$ & 0.359 & $5(20.8)$ & $46(21.7)$ \\
\hline Arthritis & $7(6.0)$ & $3(4.2)$ & 0.603 & $3(12.5)$ & $13(6.1)$ \\
\hline Puerperal sepsis & $7(6.0)$ & $1(1.4)$ & 0.132 & $0(0)$ & $8(3.8)$ \\
\hline Any abscess & $17(14.5)$ & $6(8.5)$ & 0.217 & $2(8.3)$ & $25(11.8)$ \\
\hline Endocarditis & $1(0.9)$ & $0(0)$ & 0.435 & $0(0)$ & $1(0.5)$ \\
\hline Meningitis & $2(1.7)$ & $0(0)$ & 0.271 & $0(0)$ & $2(0.9)$ \\
\hline${ }^{\mathrm{j}}$ Other microbiological finding & $20(17.1)$ & $14(19.7)$ & 0.650 & $4(16.7)$ & $38(17.9)$ \\
\hline \multicolumn{6}{|l|}{ Disease severity } \\
\hline${ }^{\mathrm{k}}$ Hypotension & $43(36.8)$ & $20(28.2)$ & 0.033 & $5(20.8)$ & $68(32.1)$ \\
\hline${ }^{1} \mathrm{ICU}$ admission & $34(29.1)$ & $8(11.3)$ & 0.005 & $4(16.7)$ & $46(21.7)$ \\
\hline${ }^{\mathrm{m}}$ STSS & $22(18.8)$ & $5(7.0)$ & 0.026 & $6(25.0)$ & 33 (15.6) \\
\hline${ }^{\mathrm{n}} \mathrm{DIC}$ & $14(12.0)$ & $2(2.8)$ & 0.029 & $3(12.5)$ & $19(9.0)$ \\
\hline${ }^{\circ}$ Any surgical intervention & $50(42.7)$ & $19(26.8)$ & 0.028 & $1(4.2)$ & $70(33.0)$ \\
\hline \multicolumn{6}{|l|}{ Mortality } \\
\hline In 7 days & $1(0.9)$ & $3(4.2)$ & 0.121 & $10(41.7)$ & $14(6.6)$ \\
\hline In 30 days & $6(5.1)$ & $6(8.5)$ & 0.366 & $11(45.8)$ & $23(10.8)$ \\
\hline In 90 days & $7(6.0)$ & $8(11.3)$ & 0.195 & $13(54.2)$ & $28(13.2)$ \\
\hline
\end{tabular}

IDSC Infectious disease specialist consultation, NADC No available data on consultation, IVDU Intravenous drug user, SSTI Skin and soft tissue infections, ICU Intensive care unit, STSS Streptococcal toxic shock syndrome, DIC Disseminated intravascular coagulation

Data represent: No, (\%) of the group

${ }^{a} p$ value: comparison of the groups IDSC+ and IDSC - with chi-square test expect with two-sample for age

${ }^{\mathrm{b}}$ Includes atherosclerotic cardiovascular or cerebrovascular disease, peripheral arterial disease, and transient ischemic attack diagnosed by neurologist

${ }^{\mathrm{c}}$ Includes asthma, chronic obstructive pulmonary disease, lung fibrosis, and chronic hypoventilation

${ }^{\mathrm{d}}$ Includes alcohol abuse or alcohol-related medical or social problem according the patient records

${ }^{\mathrm{e}}$ Includes use of $\geq 5 \mathrm{mg}$ of prednisolon last $28 \mathrm{~d}$ or biological medicine/cytotoxic drugs last 1 year

${ }^{\mathrm{f}}$ Includes leukemia, lymphoma, solid tumors, and other cancers

${ }^{g}$ None of chronic diseases above, nor chronic kidney or liver disease, heart failure, inflammatory bowel disease, hypertension, or hypercholesterolemia

${ }^{\mathrm{h}}$ Includes erysipelas, cellulitis, infected ulcers, wound infections

${ }^{\mathrm{i}}$ Definition: intraoperative diagnosis of NF made by specialists in the fields of surgery

${ }^{\mathrm{j}}$ Includes any other culture positive finding than GAS in clinical sample until the fifth day after taking the blood cultures. Excludes probable skin contaminants and mixed flora

${ }^{\mathrm{k}}$ Systolic blood pressure $<100 \mathrm{mmHg}$ when the positive blood cultures for GAS were taken

${ }^{1}$ Admission to the ICU during the $48 \mathrm{~h}$ after the positive blood cultures for GAS

${ }^{\mathrm{m}}$ As defined in the methods section

${ }^{\mathrm{n}}$ Definition: clinical picture (bleeding, thrombus, petechiae) and thrombocyte count $<100 \times 109 / \mathrm{L}$ or abnormal value of INR (international normalized ratio), APTT (activated partial thromboplastin time), TT (thrombin time), or FIDD (fibrin D-dimer)

${ }^{\mathrm{o}}$ Includes interventions ranging from wound revision or abscess drainage to amputation 
Table 2 Emm-type distribution of bacteremic GAS isolates $(n=212)$ included in the study

\begin{tabular}{|c|c|c|c|c|c|c|c|c|}
\hline \multirow[t]{2}{*}{ Year } & \multicolumn{8}{|c|}{ Number of isolates ( $\%$ of total) per year } \\
\hline & emm1 & emm4 & emm12 & emm28 & emm89 & Others & Missing & Total \\
\hline 2007 & $4(30.8)$ & $0(0)$ & $1(7.70)$ & $2(15.4)$ & $0(0)$ & $5(38.5)$ & $1(7.7)$ & 13 \\
\hline 2008 & $4(33.3)$ & $0(0)$ & $1(8.3)$ & $4(33.3)$ & $0(0)$ & $3(13.2)$ & $0(0)$ & 12 \\
\hline 2009 & $1(7.1)$ & $3(21.4)$ & $1(7.1)$ & $2(14.3)$ & $2(14.3)$ & $4(28.6)$ & $1(7.1)$ & 14 \\
\hline 2010 & $0(0)$ & $0(0)$ & $0(0)$ & $2(50.0)$ & $0(0)$ & $2(50.0)$ & $0(0)$ & 4 \\
\hline 2011 & $0(0)$ & $3(27.3)$ & $1(9.1)$ & $1(9.1)$ & $4(36.4)$ & $2(18.2)$ & $0(0)$ & 11 \\
\hline 2012 & $2(10.0)$ & $1(5.0)$ & $1(5.0)$ & $3(15.0)$ & $10(50.0)$ & $3(15.0)$ & $0(0)$ & 20 \\
\hline 2013 & $3(10.1)$ & $1(3.3)$ & $1(3.3)$ & $8(26.7)$ & $12(40.0)$ & $5(16.7)$ & $0(0)$ & 30 \\
\hline 2014 & $1(12.5)$ & $1(12.5)$ & $0(0)$ & $0(0)$ & $3(37.5)$ & $3(37.5)$ & $0(0)$ & 8 \\
\hline 2015 & $0(0)$ & $0(0)$ & $0(0)$ & $6(42.9)$ & $6(42.9)$ & $2(14.3)$ & $0(0)$ & 14 \\
\hline 2016 & $4(22.2)$ & $2(11.1)$ & $5(27.8)$ & $4(22.2)$ & $0(0)$ & $3(16.7)$ & $0(0)$ & 18 \\
\hline 2017 & $6(21.4)$ & $1(3.6)$ & $6(21.4)$ & $11(39.3)$ & $2(7.1)$ & $2(7.1)$ & $0(0)$ & 28 \\
\hline 2018 & $17(42.5)$ & $2(5.0)$ & $1(2.5)$ & $15(37.5)$ & $1(2.5)$ & $4(10.0)$ & $0(0)$ & 40 \\
\hline total & $42(19.8)$ & $14(6.6)$ & $18(8.5)$ & $58(27.4)$ & 40 (18.9) & 38 (17.9) & $2(0.9)$ & 212 \\
\hline
\end{tabular}

already in 2016 and 2017 when also the incidence of GAS bacteremia began to increase.

The emm types observed in ICU treated cases $(n=46)$ in descending order were as follows: emm $28(17,37.0 \%)$, emm 1 (9, 19.6\%), emm89 (7, 15.2\%), emm 12 (6, 13.0\%), and emm 4 $(1,2.20 \%)$ and other emm types $(6,13.0 \%)$. In cases meeting the STSS criteria $(n=33)$, the most prevalent emm types were emm 28 (9, 27.3\%), emm 1 (8, 24.2\%), emm 89 (5, 15.2\%), and emm $12(4,12.1 \%)$. The emm-type distribution of cases with $\mathrm{NF}(n=6)$ was emm 1 [2], emm89 [2], emm 12 [1], and emm6.4 [1].

\section{Antimicrobial susceptibility}

All GAS strains were susceptible to benzylpenicillin. Resistance rate to clindamycin was $4.2 \%$ (9 cases) and $2.4 \%$ (5 cases) were intermediately sensitive to clindamycin. Erythromycin sensitivity was not investigated in laboratory in years 2011-2016. Erythromycin resistance rate was 1.4\% (3 cases in year 2018) and 5.2\% (11 cases during the years 2009,2017 , and 2018) were intermediately sensitive to erythromycin. Antimicrobial susceptibility did not differ significantly between study groups.

\section{Infectious diseases specialist consultation and antibiotic treatment selection}

The percentages of the selected first-line antibiotics and adjunctive antibiotics in the treatment of GAS bacteremia stratified by IDSC are shown in Figs. 2 and 3. The most commonly used first-line antibiotics were penicillin G and iv-cephalosporins. The selection of first-line antibiotic differed significantly between the groups IDSC+ and IDSC- (chi-square test, $p<0.001$ ). Among IDSC+ cases, $57.3 \%$ were on penicillin $\mathrm{G}$ treatment whereas in the group IDSC- only $22.5 \%$, respectively $(\mathrm{OR}=4.61,95 \%$ CI $2.37-8.97 ; p<0.001)$. Among IDSC+ cases only $23.1 \%$ were on iv-cephalosporins treatment whereas in the IDSC - group up to $50.7 \%(\mathrm{OR}=0.29,95 \% \mathrm{CI}$ $0.16-0.55 ; p<0.001)$. The most commonly used adjunctive antibiotics were clindamycin, fluoroquinolones, and metronidazole. The use of any adjunctive antibiotics was significantly less common in the group IDSC- $(39.4 \%)$ than IDSC+ $(66.7 \%)$ (chi-square test, $p<0.001$ ). The use of clindamycin as adjunctive antibiotic was more common among IDSC+ cases $(54.7 \%)$ than IDSC $-(21.1 \%)(\mathrm{OR}=4.51,95 \% \mathrm{CI}$ $2.29-8.87 ; p<0.001)$.

The antibiotic treatment selection was also viewed in two time periods, 2007-2012 and 2013-2018. In the whole study population, the use of penicillin $\mathrm{G}$ as first-line antibiotic increased during the study period from $23.0 \%$ (2007-2012) to 47.8\% (2013-2018) $(p<0.001)$.

\section{The antibiotic treatment selections in ICU}

Table 3 summarizes the antibiotic treatment selection in the ICU-treated cases compared with the non-ICU-treated cases with or without IDSC. The ICU-treated cases were analyzed as one cohort because 34 cases ( $81 \%$ ) obtained IDSC. Cases with NADC (see Introduction; definitions) were excluded from this analysis. In 12 cases, the antibiotic treatment included 3 antibiotics. Eight of these 12 were ICU-treated. In 8 cases, the third antibiotic was regarded as clindamycin while the second antibiotic was regarded as either a carbapenem or a fluoroquinolone combined to the first-line antibiotic penicillin during the data collection. In ICU-treated cases, the use of 
Fig. 2 The percentages of the selected first-line antibiotics in the treatment of GAS bacteremia stratified by IDSC. $* p<0.001$ (binary logistic regression)

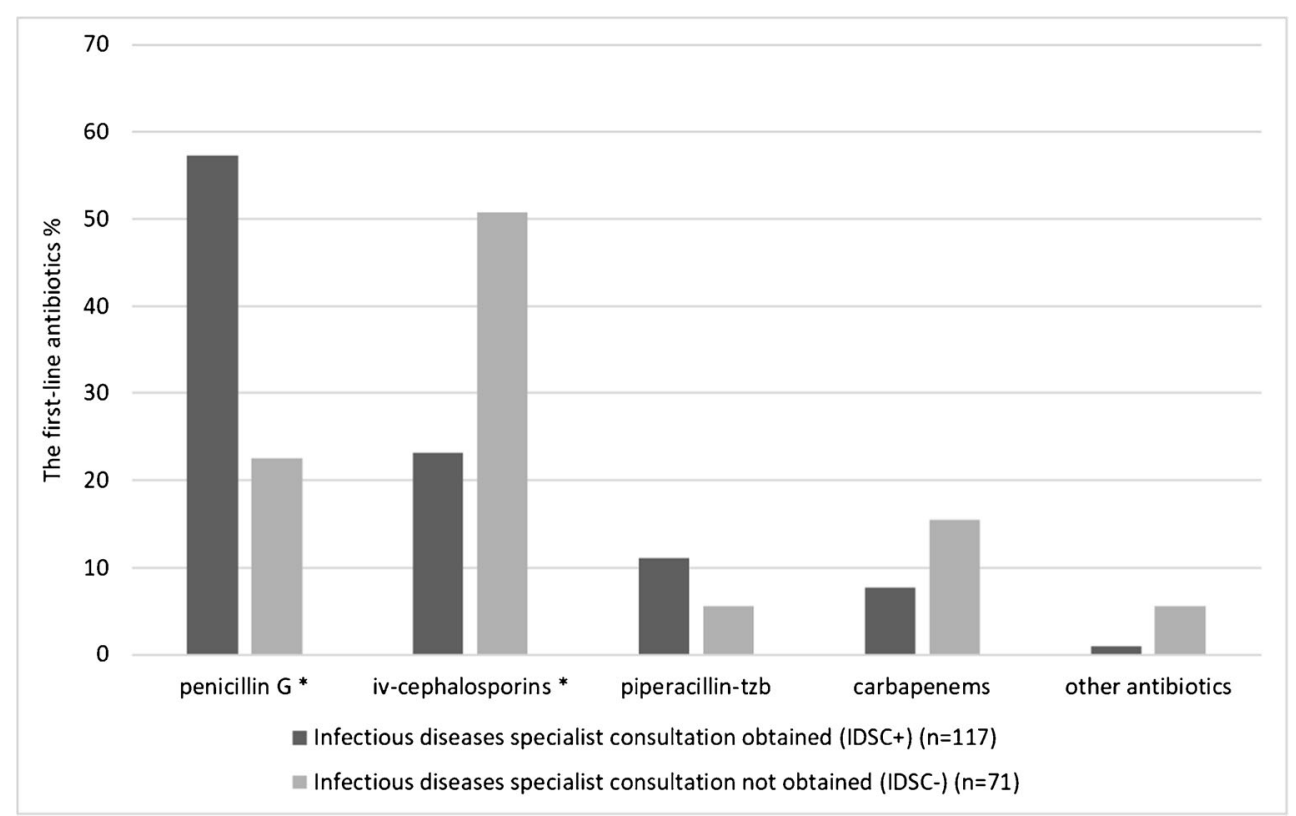

clindamycin and carbapenems were more prevalent than in non-ICU-treated.

\section{Discussion}

This study shows an overview of GAS bacteremia in one hospital district in Finland during 2007-2018. We observed the alarmingly high incidence of GAS bacteremia in 2018, the increasing trend in incidence during the study period, and that IDSC increased the use of narrow-spectrum beta-lactam antibiotics, but, on the other hand, increased also the use of clindamycin as adjunctive antibiotic.

Our observed overall incidence of GAS bacteremia is comparable with earlier studies from Finland with iGAS incidence ranging from $2.46 / 100,000$ to $3.6 / 100,000$, but the peakincidence is higher than ever before reported from Finland $[4,5,18]$. The criteria for reporting iGAS to the NIDR have remained unaltered throughout the years. The overall incidence is also comparable with iGAS studies conducted in other western countries [18-21]. The incidence of iGAS infections increased in 2018 also in whole Finland to the highest
Fig. 3 The percentages of the selected adjunctive antibiotics in the two study groups (IDSC+ and IDSC-)

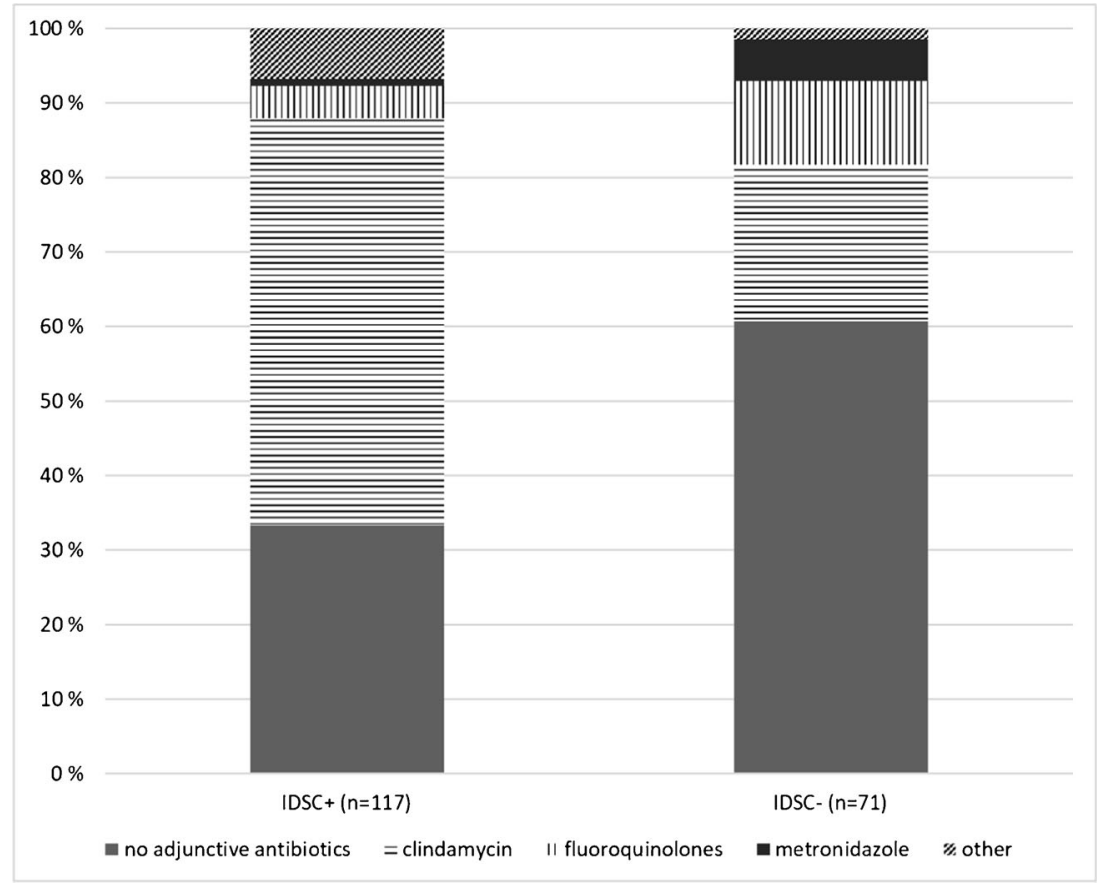


Table 3 Comparison of the antibiotic treatment selections in ICU-treated and non-ICU-treated cases with or without IDSC

\begin{tabular}{|c|c|c|c|c|c|c|c|}
\hline Antibiotic selection & $\begin{array}{l}\text { ICU treated } \\
(n=42)\end{array}$ & $\begin{array}{l}\text { Non-ICU- } \\
\text { treated } \\
\text { IDSC+ }(n=83)\end{array}$ & $\begin{array}{l}\text { OR } \\
(95 \% \mathrm{CI})\end{array}$ & $p$ value $^{\mathrm{a}}$ & $\begin{array}{l}\text { Non-ICU- } \\
\text { treated } \\
\text { IDSC- }(n=63)\end{array}$ & OR $(95 \% \mathrm{CI})$ & $p$ value $^{\mathrm{b}}$ \\
\hline Penicillin $\mathrm{G}$ as FA & $21(50.0)$ & $49(59.0)$ & $1.44(0.68-3.04)$ & 0.337 & $13(20.6)$ & $0.26(0.11-0.61)$ & 0.002 \\
\hline Iv-cephalosporins as FA & $5(11.9)$ & $23(27.7)$ & $2.84(0.99-8.11)$ & 0.052 & $35(55.6)$ & $9.25(3.21-26.64)$ & $<0.001$ \\
\hline Piperacillin-tzb as FA & $6(14.3)$ & $7(8.4)$ & $0.55(0.17-1.76)$ & 0.316 & $4(6.3)$ & $0.41(0.11-1.54)$ & 0.185 \\
\hline Carbapenems as FA & $10(23.8)$ & $3(3.6)$ & $0.12(0.031-0.47)$ & 0.002 & $7(11.1)$ & $0.40(0.14-1.15)$ & 0.090 \\
\hline Clindamycin use in all combinations $^{c}$ & $36(85.7)$ & $43(51.8)$ & $0.18(0.07-0.47)$ & $<0.001$ & $8(12.7)$ & $0.02(0.01-0.08)$ & $<0.001$ \\
\hline
\end{tabular}

IDSC infectious disease specialist consultation, $I C U$ intensive care unit, $F A$ first-line antibiotics

Data represents: No, (\%) of the group

${ }^{a} p$ value: comparison of the groups ICU-treated and non-ICU-treated IDSC+ with logistic regression

${ }^{\mathrm{b}} p$ value: comparison of the groups ICU-treated and non-ICU treated IDSC- with logistic regression

${ }^{\mathrm{c}}$ Clindamycin in all combinations includes all reported cases with clindamycin use (even as third antibiotic)

level of 6.74/100,000 ever since 1995 when NIDR was established [14]. However, as there is no common international definition on an iGAS case, and the surveillance systems differ between countries, exact comparisons on iGAS rates between countries and studies need to be done with caution. With a broader case definition (a case with blood/CSF GAS isolate, or with a positive GAS culture from any sterile site, or with clinical signs of iGAS and a GAS positive culture from a nonsterile site), we would have recorded even more cases.

The observed emm-type distribution in this study is, in general, similar to earlier studies in Finland and Europe [4, $5,22]$. The proportion of emm 1 was high in the years $2007-$ 2008 and increased again since 2016. In 2018, emm 1 was the most prevalent emm type in our HD as well as also in the whole country, thus most probably causing the epidemic wave [23]. Similarly, Lynskey et al. have reported a dominant new emm 1 Streptococcus pyogenes lineage emerging in England from year 2015 onwards [8]. Among severe GAS bacteremia cases with ICU admission, STSS and NF emm 1 was a common emm type. This is in line with earlier studies where emm 1 has been associated with death and severe manifestations such as STSS and NF $[2,21,22]$. During the study period 20072018, emm 28 has been among the three most common emm types in Finland [23]. In the current study, emm 28 was the most prevalent $\mathrm{emm}$ type. In our study, a surprisingly high proportion of ICU-treated and STSS cases were caused by emm28. In a previous large study from Europe, only $9 \%$ of STSS cases were caused by emm 28 [22]. Latronico et al. reported a new emm 89 clone emerging in 2009 and spreading rapidly in Finland [24]. This was also observed in HDSWF in years 2011-2013.

The prevalence of diabetes and malignancies as underlying condition was comparable with other studies from Northern Europe [25-27]. Even higher prevalence of diabetes (29.3\%) and association with iGAS disease and death were observed in a recent study from the USA [28]. The rates of STSS cases in our study (15.6\%) were comparable to rates 10.0 to $19.0 \%$ that were observed in other studies from western countries [18, 25, 26, 28, 29]. However, in our study, the CFR for STSS in 90 days $(27.3 \%)$ was lower than in other studies with CFR $38-44 \%[18,21]$. The study population with STSS in this study was, of course, limited.

In the current study, IDSC increased the use of penicillin G as first-line antibiotic whereas the lack of IDSC increased the use of broad-spectrum beta-lactam antibiotics. We also discovered that the use of penicillin $\mathrm{G}$ in whole study population increased significantly during the later study period (20122018). This observation is important because the global concern of antimicrobial resistance calls for action. The first generally available antimicrobial guide of the HDSWF was published in the end of 2012. The amount of infectious diseases specialists has increased during the later study period (20122018) in HDSWF. These may explain, at least partly, the improved adherence to the rational use of antibiotics. Seppälä et al have earlier shown that nationwide guidance on antibiotic use resulted in reduction in the use of macrolide antibiotics for outpatient respiratory and skin infections and a significant decline in the frequency of erythromycin resistance among group A streptococci [30]. However, the latter may be confounded by variation of the prevalent emm types, as shown by Smit et al. [5].

To our knowledge there are no earlier studies regarding the role of IDSC in the antibiotic treatment selection of GAS bacteremia. However, many previous studies have demonstrated the benefits of IDSC in Staphylococcus aureus infections [31-33].

According to literature, the use of clindamycin as adjunctive therapy for non-STSS iGAS infections is elusive whereas the benefit is observed in cases with STSS and NF [11-13]. Brindle et al. showed in a clinical trial that the addition of clindamycin to flucloxacillin did not improve outcome in limb cellulitis but doubled the likelihood of diarrhea [34]. Some 
international guidelines recommend considering addition of clindamycin to beta-lactam therapy in GAS bacteremia without shock or other organ failure [35]. The rationale behind this suggestion is based on a small retrospective study in children as well as on experimental mouse model data [36, 37]. There seems to be conflicting opinions on the indications of the combined clindamycin use internationally. In the current study, the use of clindamycin as adjunctive antibiotic was more common than the prevalence of STSS or NF, and it occurred mainly in the group IDSC+. Among ICU-treated cases, the clindamycin use was most prevalent. However, the use of clindamycin as adjunctive therapy for non-severe iGAS infections lacks evidence of clinical trials with adult patients and is known to cause high risk for adverse consequences and the use should thus be avoided in these cases.

Any other culture positive finding than GAS in a clinical sample was equally present in the study groups. Thus, polymicrobial findings do not explain the excessive use of cephalosporins in the group IDSC- nor the excessive clindamycin use in the group IDSC+.

The strength of the current study is that the clinical data were gathered by reading all the electronic patient records by an infectious diseases specialist. The electronic patient records offered detailed data on, e.g., antimicrobial therapy. This study has also limitations. The study population is small and represents patients only from one hospital district in Finland. Regarding antimicrobial therapy and its side-effects, we did not study the length of clindamycin use or the prevalence of diarrhea. On average, the consultations regarding the antibiotic selections in bacteremic patients, even done by phone, are well documented in HDSWF, but as the study method is retrospective, there is a possibility that undocumented IDSC took also place. Infectious diseases specialist visits regularly in ICU and takes care of the patient with the physicians of the ICU. These routine consultations taking place during the rounds are not necessarily always documented. Thus, the proportion of IDSC in ICU may be even higher than reported in this study.

In conclusion, GAS caused bacteremias with alarmingly high incidence in one hospital district in Finland in 2018. Narrow-spectrum beta-lactam antibiotics were chosen, but excess clindamycin as adjunctive antibiotic was used, if IDSC took place. This highlights the importance of availability of IDSC when aiming for more rational antibiotic use but calls for improved practice among infectious diseases specialists by avoiding combination therapy with clindamycin in non-severe iGAS infections.

Acknowledgements We thank Mirva Virolainen and Mari Virta for technical support with emm typing and Esa Rintala for assistance with the SAI database and NIDR and advises on the manuscript. We also acknowledge Teemu Möttönen and Jan-Erik Löflund for providing the data on population statistics.
Authors' contributions and declaration The material is original and has not been published elsewhere. Parts of this study were presented as a poster at the Annual meeting of the Nordic Society of Clinical Microbiology and Infectious Diseases (NSCMID) in Trondheim, Norway, 2019. All authors have made substantive intellectual contributions to the study conception, design and manuscript and approved the final version for submission and are able to account for its content.

Funding information Open access funding provided by University of Turku (UTU) including Turku University Central Hospital. This work was supported by the Maud Kuistila Foundation (2018-0063F to JVi), The Finnish Medical Foundation (2018-2136 to JVi), the Rauno ja Anne Puolimatka Foundation (2017 to JVi), and the Competitive State Financing of the Expert Responsibility area of Turku University Hospital (13285 to JVu and 13604 to JO) and the Academy of Finland (308482 to JVu).

Data availability The datasets generated during the current study are not publicly available as they contain health related data but limited datasets (without any identifiable, person-related data) are available from the corresponding author on reasonable request.

\section{Compliance with ethical standards}

Conflict of interest JVi reports a travel grant from MSD, Gilead, and Nordic Society of Clinical Microbiology and Infectious Disease and lecture fee from MSD and the Finnish Medical Society Duodecim. JVu reports receiving lecture fees or travel support as lecturer from nonprofit organizations (Nordic Society of Clinical Microbiology and Infectious Disease, the Finnish Society of Infectious Disease Research and the Finnish Medical Society Duodecim). JO has been a scientific advisor (review panel or advisory committee) to Gilead Sciences Finland, GlaxoSmithKline, MSD Finland, and Unimedic Pharma AB, received lecture honoraria from Gilead Sciences Finland, GlaxoSmithKline, MSD Finland, and received coverage for congress travel/accommodation expenses from Gilead, Grifols, and MSD. TV, $\mathrm{KGYH}$, and KRJ report no conflict of interest.

Ethical approval This registry study was approved by the Turku Clinical Research Centre.

Open Access This article is licensed under a Creative Commons Attribution 4.0 International License, which permits use, sharing, adaptation, distribution and reproduction in any medium or format, as long as you give appropriate credit to the original author(s) and the source, provide a link to the Creative Commons licence, and indicate if changes were made. The images or other third party material in this article are included in the article's Creative Commons licence, unless indicated otherwise in a credit line to the material. If material is not included in the article's Creative Commons licence and your intended use is not permitted by statutory regulation or exceeds the permitted use, you will need to obtain permission directly from the copyright holder. To view a copy of this licence, visit http://creativecommons.org/licenses/by/4.0/.

\section{References}

1. Carapetis JR, Steer AC, Mulholland EK, Weber M (2005) The global burden of group A streptococcal diseases. Lancet Infect Dis 5(11):685-694. https://doi.org/10.1016/S1473-3099(05) 70267-X 
2. O'Loughlin RE, Roberson A, Cieslak PR, Lynfield R, Gershman K, Craig A et al (2007) The epidemiology of invasive group A streptococcal infection and potential vaccine implications: United States, 2000-2004. Clin Infect Dis 45(7):853-862. https://doi.org/10.1086/ 521264

3. Siljander T, Toropainen M, Muotiala A, Hoe NP, Musser JM, Vuopio-Varkila J (2006) Emm typing of invasive T28 group A streptococci, 1995-2004, Finland. J Med Microbiol 55(Pt 12): 1701-1706. https://doi.org/10.1099/jmm.0.46690-0

4. Siljander T, Lyytikäinen O, Vähäkuopus S, Snellman M, Jalava J, Vuopio J (2010) Epidemiology, outcome and emm types of invasive group A streptococcal infections in Finland. Eur J Clin Microbiol Infect Dis 29(10):1229-1235. https://doi.org/10.1007/ s10096-010-0989-9

5. Smit PW, Lindholm L, Lyytikäinen O, Jalava J, Pätäri-Sampo A, Vuopio J (2015) Epidemiology and emm types of invasive group A streptococcal infections in Finland, 2008-2013. Eur J Clin Microbiol Infect Dis 34(10):2131-2136. https://doi.org/10.1007/ s10096-015-2462-2

6. Williamson DA, Morgan J, Hope V, Fraser JD, Moreland NJ, Proft T et al (2015) Increasing incidence of invasive group A streptococcus disease in New Zealand, 2002-2012: a national populationbased study. J Inf Secur 70(2):127-134. https://doi.org/10.1016/j. jinf.2014.09.001

7. Darenberg J, Henriques-Normark B, Lepp T, Tegmark-Wisell K, Tegnell A, Widgren K (2013) Increased incidence of invasive group A streptococcal infections in Sweden, January 2012-February 2013. Euro Surveill 18(14):20443. https://doi.org/10.2807/15607917.es2013.18.14.20443

8. Lynskey NN, Jauneikaite E, Li HK, Zhi X, Turner CE, Mosavie M et al (2019) Emergence of dominant toxigenic M1T1 Streptococcus pyogenes clone during increased scarlet fever activity in England: a population-based molecular epidemiological study. Lancet Infect Dis 19(11):1209-1218. https://doi.org/10.1016/S1473-3099(19) 30446-3

9. Vannice K, Ricaldi J, Nanduri S, Fang FC, Lynch J, Bryson-Cahn C et al (2019) Streptococcus pyogenes pbp2x mutation confers reduced susceptibility to beta-lactam antibiotics. Clin Infect Dis. https://doi.org/10.1093/cid/ciz1000

10. Musser JM, Beres SB, Zhu L, Olsen RJ, Vuopio J, Hyyryläinen H et al (2020) Reduced in vitro susceptibility of Streptococcus pyogenes to beta-lactam antibiotics associated with mutations in the pbp2x gene is geographically widespread. J Clin Microbiol. https://doi.org/10.1128/JCM.01993-19

11. Carapetis JR, Jacoby P, Carville K, Ang SJ, Curtis N, Andrews R (2014) Effectiveness of clindamycin and intravenous immunoglobulin, and risk of disease in contacts, in invasive group a streptococcal infections. Clin Infect Dis 59(3):358-365. https://doi.org/10. 1093/cid/ciu304

12. Linnér A, Darenberg J, Sjölin J, Henriques-Normark B, NorrbyTeglund A (2014) Clinical efficacy of polyspecific intravenous immunoglobulin therapy in patients with streptococcal toxic shock syndrome: a comparative observational study. Clin Infect Dis 59(6):851-857. https://doi.org/10.1093/cid/ciu449

13. Andreoni F, Zürcher C, Tarnutzer A, Schilcher K, Neff A, Keller N et al (2017) Clindamycin affects group A streptococcus virulence factors and improves clinical outcome. J Infect Dis 215(2):269277. https://doi.org/10.1093/infdis/jiw229

14. The Finnish institute of health and welfare (THL). The National Infectious Disease Register (NIDR). S.pyogenes. Available at: https://sampo.thl.fi/pivot/prod/fi/ttr/shp/fact_shp?row=area$12260 \&$ column$=$ time $-12059 \&$ filter=reportgroup -12272 . Accessed November 2019

15. Centers for Disease Control and Prevention. Protocol for emm typing. Available at: https://www.cdc.gov/streplab/groupa-strep/emmtyping-protocol.html. Accessed November 2019
16. Charlson ME, Pompei P, Ales KL, MacKenzie CR (1987) A new method of classifying prognostic comorbidity in longitudinal studies: development and validation. J Chronic Dis 40(5):373-383. https://doi.org/10.1016/0021-9681(87)90171-8

17. Centers for Disease Control and Prevention. National Notifiable Diseases Surveillance System (NNDS). Streptococcal Toxic Shock Syndrome 2010 Case definition. Available at: https:/ wwwn.cdc.gov/nndss/conditions/streptococcal-toxic-shocksyndrome/case-definition/2010/. Accessed November 2019

18. Lamagni TL, Darenberg J, Luca-Harari B, Siljander T, Efstratiou A, Henriques-Normark B et al (2008) Epidemiology of severe Streptococcus pyogenes disease in Europe. J Clin Microbiol 46(7):2359-2367. https://doi.org/10.1128/JCM.00422-08

19. Naseer U, Steinbakk M, Blystad H, Caugant DA (2016) Epidemiology of invasive group a streptococcal infections in Norway 2010-2014: a retrospective cohort study. Eur J Clin Microbiol Infect Dis 35(10):1639-1648. https://doi.org/10.1007/ s10096-016-2704-y

20. Laupland KB, Pasquill K, Parfitt EC, Steele L (2019) Bloodstream infection due to $\beta$-hemolytic streptococci: a population-based comparative analysis. Infection 47(6):1021-1025. https://doi.org/10. 1007/s15010-019-01356-9

21. Nelson GE, Pondo T, Toews K, Farley MM, Lindegren ML, Lynfield R et al (2016) Epidemiology of invasive group A streptococcal infections in the United States, 2005-2012. Clin Infect Dis 63(4):478-486. https://doi.org/10.1093/cid/ciw248

22. Luca-Harari B, Darenberg J, Neal S, Siljander T, Strakova L, Tanna A et al (2009) Clinical and microbiological characteristics of severe Streptococcus pyogenes disease in Europe. J Clin Microbiol 47(4): 1155-1165. https://doi.org/10.1128/JCM.02155-08

23. The Finnish institute of health and welfare (THL). The National Infectious Disease Register (NIDR). Annual reports 2010-2018: group A streptococcus. Available at: https://thl.fi/fi/web/ infektiotaudit/seuranta-ja-epidemiat/tartuntatautirekisteri/ tartuntataudit-suomessa-vuosiraportit. Accessed November 2019

24. Latronico F, Nasser W, Puhakainen K, Ollgren J, Hyyryläinen H, Beres SB et al (2016) Genomic characteristics behind the spread of bacteremic group A streptococcus type emm89 in Finland, 20042014. J Infect Dis 214(12):1987-1995. https://doi.org/10.1093/ infdis/jiw468

25. Ekelund K, Skinhøj P, Madsen J, Konradsen HB (2005) Invasive group A, B, C and G streptococcal infections in Denmark 19992002: epidemiological and clinical aspects. Clin Microbiol Infect 11(7):569-576. https://doi.org/10.1111/j.1469-0691.2005.01169.x

26. Kittang BR, Bruun T, Langeland N, Mylvaganam H, Glambek M, Skrede $S$ (2011) Invasive group A, C and G streptococcal disease in western Norway: virulence gene profiles, clinical features and outcomes. Clin Microbiol Infect 17(3):358-364. https://doi.org/10. 1111/j.1469-0691.2010.03253.x

27. Rantala S, Vuopio-Varkila J, Vuento R, Huhtala H, Syrjänen J (2009) Clinical presentations and epidemiology of betahaemolytic streptococcal bacteraemia: a population-based study. Clin Microbiol Infect 15(3):286-288. https://doi.org/10.1111/j. 1469-0691.2008.02672.x

28. Langley G, Hao Y, Pondo T, Miller L, Petit S, Thomas A et al (2016) The impact of obesity and diabetes on the risk of disease and death due to invasive group A streptococcus infections in adults. Clin Infect Dis 62(7):845-852. https://doi.org/10.1093/cid/ civ1032

29. Meehan M, Murchan S, Gavin PJ, Drew RJ, Cunney R (2018) Epidemiology of an upsurge of invasive group A streptococcal infections in Ireland, 2012-2015. J Inf Secur 77(3):183-190. https://doi.org/10.1016/j.jinf.2018.05.010

30. Seppälä H, Klaukka T, Vuopio-Varkila J, Muotiala A, Helenius H, Lager K et al (1997) The effect of changes in the consumption of macrolide antibiotics on erythromycin resistance in group a 
streptococci in Finland. Finnish study group for antimicrobial resistance. N Engl J Med 337(7):441-446. https://doi.org/10.1056/ NEJM199708143370701

31. Honda H, Krauss MJ, Jones JC, Olsen MA, Warren DK (2010) The value of infectious diseases consultation in Staphylococcus aureus bacteremia. Am J Med 123(7):631-637. https://doi.org/10.1016/j. amjmed.2010.01.015

32. Forsblom E, Kakriainen A, Ruotsalainen E, Järvinen A (2018) Methicillin-sensitive Staphylococcus aureus bacteremia in aged patients: the importance of formal infectious specialist consultation. Eur Geriatr Med 9(3):355-363. https://doi.org/10.1007/s41999018-0038-2

33. Rieg S, Peyerl-Hoffmann G, de With K, Theilacker C, Wagner D, Hübner J et al (2009) Mortality of S. aureus bacteremia and infectious diseases specialist consultation-a study of 521 patients in Germany. J Inf Secur 59(4):232-239. https://doi.org/10.1016/j. jinf.2009.07.015

34. Brindle R, Williams OM, Davies P, Harris T, Jarman H, Hay AD et al (2017) Adjunctive clindamycin for cellulitis: a clinical trial comparing flucloxacillin with or without clindamycin for the treatment of limb cellulitis. BMJ Open 7(3):e013260. https://doi. org/10.1136/bmjopen-2016-013260

35. UpToDate. The evidence-based clinical decision support resource from Wolters Kluwer. Invasive group A streptococcal infection and toxic shock syndrome: Treatment and prevention. Available at: https://www.uptodate.com/contents/invasive-group-astreptococcal-infection-and-toxic-shock-syndrome-treatment-andprevention. Accessed November 2019

36. Stevens DL, Gibbons AE, Bergstrom R, Winn V (1988) The eagle effect revisited: efficacy of clindamycin, erythromycin, and penicillin in the treatment of streptococcal myositis. J Infect Dis 158(1): 23-28. https://doi.org/10.1093/infdis/158.1.23

37. Zimbelman J, Palmer A, Todd J (1999) Improved outcome of clindamycin compared with beta-lactam antibiotic treatment for invasive Streptococcus pyogenes infection. Pediatr Infect Dis J 18(12):1096-1100. https://doi.org/10.1097/00006454-19991200000014

Publisher's note Springer Nature remains neutral with regard to jurisdictional claims in published maps and institutional affiliations. 